\title{
The Social Behavior and the Evolution of Sexually Transmitted Diseases
}

\author{
Sebastián Gonçalves ${ }^{1, \text { 冈 }}$ and Marcelo Kuperman², † \\ ${ }^{1}$ Instituto de Física Universidade Federal do Rio Grande do Sul, \\ Caixa Postal 15051, 90501-970 Porto Alegre RS, Brazil \\ ${ }^{2}$ Centro Atómico Bariloche and Instituto Balseiro, 8400 S. C. de Bariloche, Argentina
}

(Dated: October 31, 2018)

\begin{abstract}
We introduce a model for the evolution of sexually transmitted diseases, in which the social behavior is incorporated as a determinant factor for the further propagation of the infection. The system may be regarded as a society of agents where in principle anyone can sexually interact with any other one in the population. Different social behaviors are reflected in a distribution of sexual attitudes ranging from the more conservative to the more promiscuous. This is measured by what we call the promiscuity parameter. In terms of this parameter, we find a critical behavior for the evolution of the disease. There is a threshold below what the epidemic does not occur. We relate this critical value of the promiscuity to what epidemiologist call the basic reproductive number, connecting it with the other parameters of the model, namely the infectivity and the infective period in a quantitative way. We consider the possibility of subjects be grouped in couples. In this contribution only the homosexual case is analyzed.

PACS numbers: 87.19.Xx, 87.23.Ge, 89.65.-s
\end{abstract}

\section{INTRODUCTION}

In the recent years, many mathematical models of social phenomena have been formulated in order to describe a wide variety of phenomena [1]. A particular interest was shown by the evolution of epidemic processes when the structure of the underlying society is taken into account [2, 3, 4, 5]. In many of these models the existence of a threshold value that determines the further evolution of a nucleus of infection was verified [6, 7]. On the contrary, it is possible to find other situations where the absence of threshold was shown [6]. Nevertheless, the interesting feature in all of these models is that they incorporate a social aspect not taken into account in previous epidemiological analysis. In the present work, we analyze a particular family of diseases, those sexually transmitted such as AIDS, Hepatitis B, Syphilis, etc. We will focus on a particular aspect of the society very related to the propagation of these diseases: the sexual behavior. We will consider a society composed by sexually active individuals and with different behavioral patterns ranging from the most conservative or stable one, those who only have sexual intercourse with their stable and unique mate, to the most promiscuous who are continuously willing to change their sexual partner. The dynamic of the disease will be associated to the $S I R$ case, where, at a given time, each individual in the population can be in one of the following three stages: susceptible $(S)$, infective $(I)$, and refractory or removed $(R)$. A susceptible individual can become infective through contagion by an infective individual. Once an element has been infective, it enters a cycle that, after a fixed infection time, ends when the element reaches the refractory (or removed in case of death, as in the present model) state. At this stage the individual cannot be infected again nor infect the others, therefore the infection ultimately leads to definitive removal of elements from the susceptible population. This family of models has been used to describe the dynamics of well-known infectious diseases, such as AIDS, rabies, and black death, by means of mean field approximation [8].

\section{THE MODEL}

Our model of the society conforms to a set of $N$ sexually active subjects, which in principle are grouped in couples with the exception of a controlled proportion of singles $\left(\rho_{\text {sng }}\right)$. Initially a "promiscuity" value $p_{i}$ is randomly assigned to each agent from a semi-Gaussian distribution of width $\hat{p} . p_{i}$ determines the individual's tendency to dismiss his stable mate and go out -or just to go out in the case of the singles- to look for an occasional intercourse. In terms of the model the individual promiscuity $p_{i}$ is the probability of trying to meet somebody else on each opportunity, determined by the time step of the simulation. Then, a susceptible individual can become infective, after a sexual intercourse with an infective one, with probability $\beta$. The infective individuals remain infectious for a period $\tau$, after what they are removed by death.

Every time step each individual must choose to have sex with his couple or with somebody else, the latter occurs with probability $p_{i}$. Those who have decided to go out, must chose a partner at random and if the latter has decided to go out too, the occasional couple is made. Therefore there is no social structure a priori. The web of contacts is constructed dynamically during the simulation depending on the percentage of singles and $\hat{p}$. Thus, 
a random proportion of subjects, from those who have decided to go out, are reorganized in temporary couples every time step. Those who were abandoned by their couples and decided no to look for another partner will not have sex during this time step. The same is true for the singles who decided not to go out. Eventually, an individual who goes out to look for somebody else may not have success remaining sexually inactive. In the present model, once an individual is removed, no new members are added to the society. This more complex situation will be analyzed in a further work.

Summarizing, the model has four parameters: $\rho_{s n g}, \hat{p}$, $\tau$, and $\beta$ The first one controls the proportion of singles, the second one is the width of the semi-Gaussian distribution of $p_{i}$, while the latter's two are the same for all individuals. As long as we assume that all agents are-except of the promiscuity and the marital status - identical (i.e. the infectivity between any two subjects is symmetrical), the model may be regarded as representing the spread of HIV/AIDS in a male homosexual community with no distinction between passive and active partners.

\section{NUMERICAL RESULTS}

As was stated in the Introduction our results are for fixed number $(N)$ of subjects, all sexually active -in practice an individual could be sexually low active if single and with low $p_{i}$. No new members are incorporated during the dynamics and no other cause of death apart from the sexually transmitted disease is considered. Therefore the number of susceptibles $(S)$, infectives $(I)$, and removeds $(R)$ is conserved: $N=S+I+R$. Most of the simulation are performed for 100000 subjects population with only one individual initially infective. A hundred realizations (different runs starting with a different shuffling of the $p_{i}$ among individuals) were considered to make statistics. In order to give the simulations a human dimension one time step represents a day, and we make runs up to 5yr-100yr of simulation time, depending on the selected parameters (namely $\tau$ ).

If we take for example $\rho_{\text {sng }}=0$ (all subjects paired), $\hat{p}=1 / 30, \tau=1 \mathrm{yr}$, and $\beta=1 \mathrm{day}^{-1}$, the resulting time evolution of the infectives, $I(t)$, and susceptibles, $S(t)$, is similar to what can be obtained solving the classical $S I R$ differential equations $[8]$. However, the similarities are only qualitative, because in our scheme different runs for the same parameters give different positions for the infectives maximum peak occurrence for example. Even more, a small percentage of the realizations does not produce an epidemic; i.e the infection dies out taking just few individuals. This is an unexpected result from the point of view of mean field standard continuous models, however it does not seem unreasonable at all, considering that people, behavior and randomness are involved in real life. Thus statistically there is a low but non zero

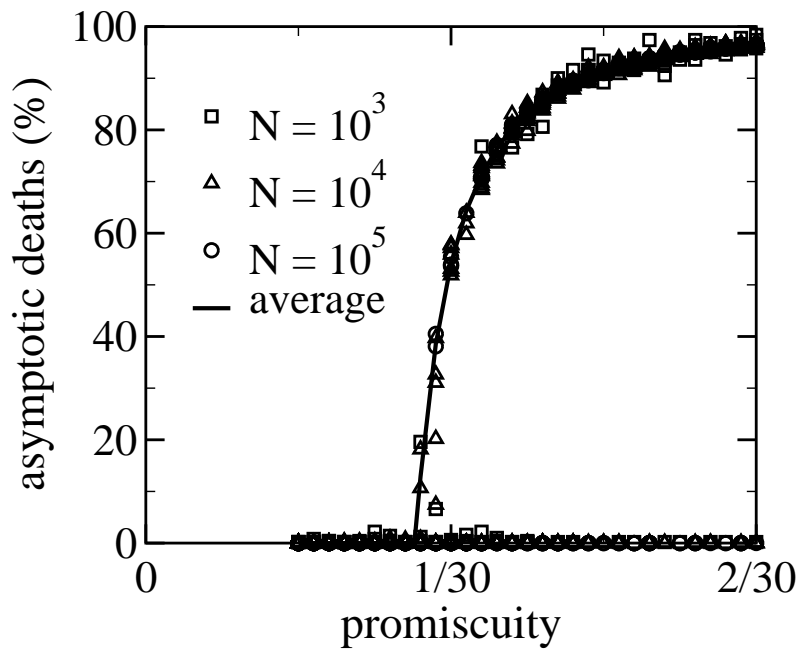

FIG. 1: Asymptotic percentage of death as a function of the promiscuity distribution width $\hat{p}$. Parameters are $\tau=1 \mathrm{yr}$ and $\beta=1$. Notice the onset of the epidemic at the critical value $\hat{p}_{c}=0.9 / 30$. Numerical results obtained with $N=1000$, 10000 , and 100000 subjects, 100 realization and for $\rho_{s n g}=0$ case (all subjects paired).

probability for the infection not to evolve with these parameters, while the standard SIR model would give an epidemic as unique, deterministic outcome. Statistically, these cases should be considered as fluctuations.

The results for several runs with the above parameters, but for $\hat{p}$ varying in the range $1 / 60-1 / 15$, and for different populations sizes are presented in Fig. 1. There it can be seen that the probability for an epidemic to occur, changes abruptly from zero to a finite value, at the critical promiscuity $\hat{p}_{c}=0.9 / 30$. We can see too that there is a non-zero probability for non-epidemic at values of $\hat{p}>\hat{p}_{c}$ as denoted by the points at the horizontal axis, showing an asymptotic very low number of death. At the same time, the asymptotic number of death increases very fast with $\hat{p}>\hat{p}_{c}$, for realizations that produce epidemics. The line in Fig. 1 is the average of asymptotic deaths calculated over all realizations which gave an epidemic (\% of asymptotic death $>1 \%$ ). In what follows we show this average instead of plotting the individuals runs. Probably, the most interesting feature found in this work -not previously reported- is the epidemic threshold in terms of the promiscuity. The critical value of the population promiscuity, $\hat{p}_{c}$, depends obviously on the values of the other parameters $\left(\tau, \beta\right.$, and $\left.p_{\text {sng }}\right)$ as we will see.

In the same Fig. 1 it can be noted that the obtained results are not an effect of the finite size of the sample. Several realizations on different sized population are plotted there, and the coincidence of all data is quite evident.

Another aspect to take into account is the possibility of the epidemic evolution of being critically affected by the number of initially infected individuals, $I(0)$. Though it is clear that for a huge proportion of initially infectives, 
the evolution will drastically change, allowing for a small increment in the number of them, namely from 1 to 5 , in samples of 10000 subjects -and even in a small population of 1000 subjects- gives the same results.

$\mathrm{Up}$ to here the results presented are for $\rho_{\text {sng }}=0$, i.e. the non singles case. How the results depend on this late parameter? Performing simulations for three different cases, i.e. $\rho_{\text {sng }}=0,0.5,1$, we can see that the qualitative behavior of the results is the same, with a displacement to higher values of $\hat{p}_{c}$ as $\rho_{\text {sng }}$ increases. The results are plotted in Fig. 2, where the shift in the value of $\hat{p}_{c}$ is observed, and also a slower evolution to high values of death as $\hat{p}$ increases. This is an expected result: once one of the members of the couple gets infective, sooner or later the other gets too; therefore, the more people are in couples, the more effective is the propagation of the epidemic. We have to remark here that in the present model the individual promiscuity is independent of the marital status.

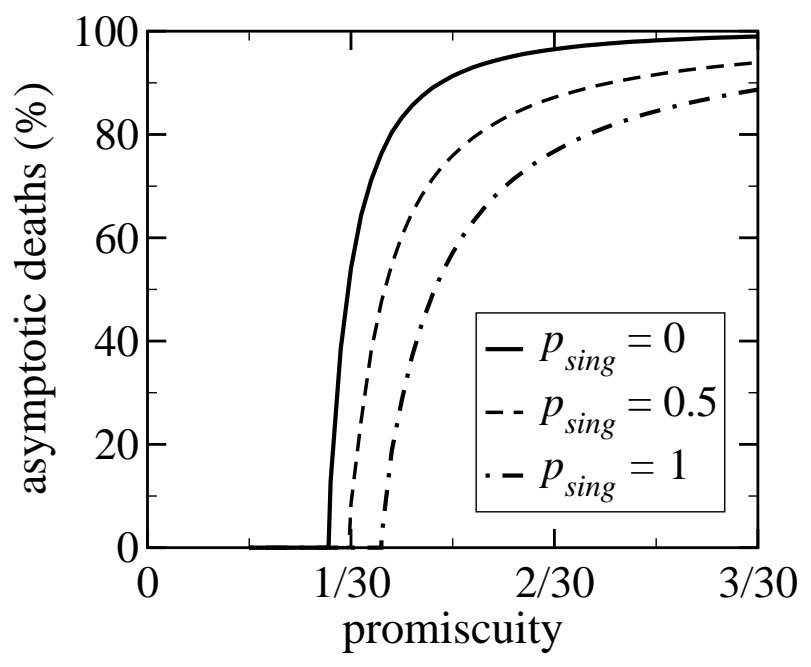

FIG. 2: Asymptotic percentage of death as a function of distribution promiscuity width for three different values of $\rho_{\text {sng }}\left(\beta=1\right.$ day $\left.^{-1}, \tau=1 \mathrm{yr}\right)$. Numerical results for 100000 subjects and 100 realizations.

It is known from classical $S I R$ models (see $\|$ ) that the relevant parameter is $\rho=a / r$, where $r$ is the infectiveness and $a$ is the removal rate. We expect to verify the same for $\beta$ and $\tau$, so the relevant parameter should be their product. This was numerically checked for different values of $\beta$ and $\tau$ confirming the collapse of all data. Consequently the critical promiscuity depends on the product of $\beta$ and $\tau$ as can be observed in Fig 3, where $\hat{p}_{c}^{2}$ as a function of $(\beta \tau)^{-1}$ is plotted along with linear fittings for the three values of $\rho_{s n g}$ studied. From that we have obtained the expression $\hat{p}_{c}^{2} \beta \tau=0.36,0.48,0.65$ (respectively for $\left.\rho_{\text {sng }}=0,0.5,1\right)$ connecting the $\hat{p}, \beta$, and $\tau$ parameters at the critical point.

As for practical purposes, we can test the threshold condition, obtained from our model, against HIV infec-

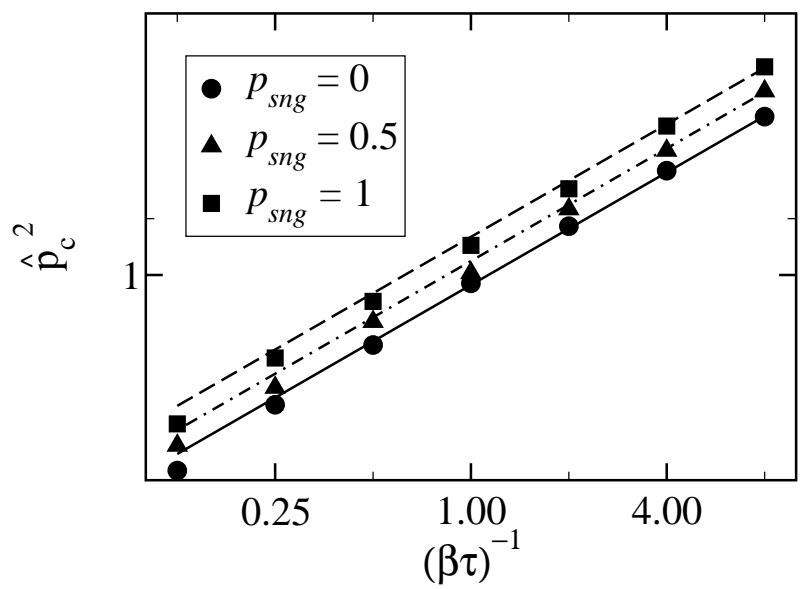

FIG. 3: Squared critical value of the distribution promiscuity width $\left(\hat{p}_{c}^{2}\right)$ as a function of the inverse product of $\beta$ and $\tau$, for three different values of $\rho_{s n g}$. Promiscuity is a per month probability, where $\beta$ is a per contact probability; $\tau$ is in years.

tion data. Assuming an infectivity $\beta=0.1$ [9] and a life time $\tau=5 y r$, we can roughly estimate $\hat{p}_{c}=0.045$, this in turn means $\left(\langle p\rangle=(2 / \pi)^{(1 / 2)} \hat{p}\right.$ for a semi-Gaussian distribution) an average critical promiscuity of $\langle p\rangle=0.036$, i.e one "excursion" every 28 days in the population average. Thus, this estimate is consistent with the outbreak of HIV infection among male homosexuals in the late 70's in the US.

So far we have considered a semi-Gaussian distribution of promiscuity. It is known today that the web of sexual contacts has a power low distribution as shown in 12 for a sample of the swedish population, so the natural question is: Are all the results presented here valid for an actual society or are they mere academic speculation with no practical application? It has been put forward for example that the scale-free structure of the sexual net make it susceptible for an epidemic to occur without any threshold [4]. In order to verify this we have repeated some runs changing the semi-Gaussian distribution by a power-law distribution:

$$
n(p)= \begin{cases}0 & \text { if } p<p_{0} \\ 2 p_{0}^{2} p^{-3} & \text { otherwise }\end{cases}
$$

and we have found again a threshold for the epidemic outbreak For this distribution the parameter $p_{0}$ has to be bigger than 0.1 for an epidemic to occur for $\beta=1$ and $\tau=1$. The results can be observed in Fig. 4, where we plot the asymptotic number of death for the power law promiscuity distribution together with the Gaussian distribution. In order to compare both distribution we have normalized the promiscuity axis to the mean population promiscuity $\langle p\rangle$. In this terms the onset of the epidemic are similar with a shift to smaller values in the power law case. Therefore the critical behavior is independent of the type of distribution. This can be explained because of the distribution cut-off; in the real world the net of contacts 
have no nodes (subjects) with infinity connectivity. In our specific model, the maximum possible promiscuity is 1 which means that the person goes out to meet someone everyday. Even in this extreme case he has a maximum for the number of possible encounters (less than 365 a year because he has to be accepted too). We think that, at the same time that our hypothesis it reasonably for a real population it gives an explanation for the apparent controversy between our results and the theoretical arguments against the existence of threshold [4].

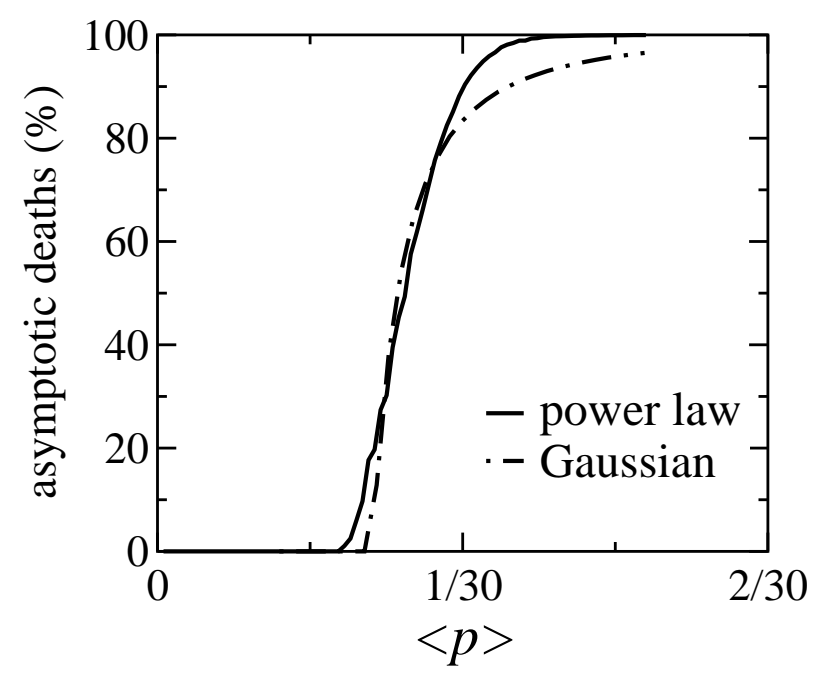

FIG. 4: Asymptotic percentage of death as a function of the mean promiscuity of the population $\langle p\rangle$ for a power-law distribution and a Gaussian distribution of promiscuity. $\mathrm{Pa}-$ rameters are $\tau=1 y r$ and $\beta=1 d a y^{-1}$. Numerical results obtained with 100000 subjects, 100 realization and for $\rho_{s n g}=0$ case (all subjects paired).

\section{CONCLUSIONS}

We have presented a model for the spread of sexual transmitted diseases, taking into account the social behavior. In spite of the simplistic way of modeling the social interactions, the model produce an outcome not previously reported in classical SIR models for infectious diseases of any kind, sexually transmitted or transmitted by other ways: a quantitative condition for the epidemic to occur. This threshold, called by epidemiologist, the basic reproductive number, was conjectured in the classical literature in an empirical way 10, 11]. Moreover, it is not predicted, nor consistent with classical $S I R$ models. On the other hand the present model gives a direct connection between disease and social parameters and the epidemic threshold. Being quantitative this connection could be of great utility to predict epidemics. The relevant parameters are four: two corresponding to epidemiological aspects and related to the disease, the probability of being infected in each sexual encounter $\beta$ and the average life time of the infectious individual $\tau$, and two related to social aspects: the distribution of sexual contacts of the population $p_{i}$, and the fraction of people that are not in stable couples. The knowledge of the last three would led us to determine the tolerated values for the infectivity $\beta$ to slow down and, why not, to stop the HIV infectious for example.

The authors thank Coordenação de Aperfeiçoamento de Pessoal de Nível Superior (CAPES, Brasil) and Secretaría de Ciencia, Tecnología e Innovación Productiva (SETCIP, Argentina) for support.

* Electronic address: sgonc@if.ufrgs.br

$\dagger$ Electronic address: kuperman@cab.cnea.gov.ar

[1] W. Weidlich. Phys. Rep. 204, 1 (1991).

[2] C. Moore and M. E. J. Newman, Phys. Rev. E 61 (1999) 5678.

[3] M. Kuperman and G. Abramson, Phys. Rev. Lett. 86, 2909 (2001).

[4] R. Pastor-Satorras and A. Vespignani, Phys. Rev. E 63, 066117 (2001).

[5] N. Zekri and J. P. Clerc, Phys. Rev. E 64, 056115 (2001).

[6] R. Pastor-Satorras and A. Vespignani, Phys. Rev. Lett. 86, 3200 (2001).

[7] M. E. J. Newman, Phys. Rev. E 66, 016128 (2002).

[8] J. D. Murray, Mathematical Biology (Springer, Berlin, 1993).

[9] T. A. Peterman et al., J. Am. med. Ass. 259, 55 (1988).

[10] R. M. May and R. M. Anderson, Phil. Trans. R. Soc. Lond. B 321, 565 (1988).

[11] R. M. May and A. L. Lloyd, Phys. Rev. E 64, 066112 (2001).

[12] F. Liljeros, C. R. Edling, L. A. Nunes Amaral, H. E. Stanley, Y. Åberg, Nature 411, 907 (2001). 